University of Nebraska - Lincoln

DigitalCommons@University of Nebraska - Lincoln

2000

\title{
Geometric Rule Learning by Clark's Nutcrackers (Nucifraga columbiana)
}

\author{
Alan Kamil \\ University of Nebraska - Lincoln, akamil1@unl.edu \\ Juli E. Jones \\ University of Nebraska - Lincoln
}

Follow this and additional works at: https://digitalcommons.unl.edu/bioscibehavior

Part of the Behavior and Ethology Commons

Kamil, Alan and Jones, Juli E., "Geometric Rule Learning by Clark's Nutcrackers (Nucifraga columbiana)" (2000). Papers in Behavior and Biological Sciences. 22.

https://digitalcommons.unl.edu/bioscibehavior/22

This Article is brought to you for free and open access by the Papers in the Biological Sciences at DigitalCommons@University of Nebraska - Lincoln. It has been accepted for inclusion in Papers in Behavior and Biological Sciences by an authorized administrator of DigitalCommons@University of Nebraska - Lincoln. 
Published in Journal of Experimental Psychology: Animal Behavior Processes 26:4 (2000), pp. 439-453; doi: 10.1037/0097-7403.26.4.439

Copyright $@ 2000$ American Psychological Association. Used by permission. "This article may not exactly replicate the final version published in the APA journal. It is not the copy of record." http://www.apa.org/journals/xan/

This research was supported by National Science Foundation Grant IBN 94-21807. We thank Brett Gibson,

Reuven Dukas, Alan Bond, and Ken Cheng for their comments on a draft of this article.

Submitted January 22, 1999; revised May 1, 2000; accepted May 1, 2000.

\title{
Geometric Rule Learning by Clark's Nutcrackers (Nucifraga columbiana)
}

\author{
Alan C. Kamil and Juli E. Jones \\ School of Biological Sciences, University of Nebraska-Lincoln \\ Corresponding author - Alan C. Kamil, School of Biological Sciences, Manter Hall, \\ University of Nebraska-Lincoln, Lincoln, Nebraska 68588-0118; email akamil@unlserve.unl.edu \\ Juli E. Jones is now at the Neuroscience and Behavior Program, University of Massachusetts.
}

\begin{abstract}
Clark's nutcrackers (Nucifraga columbiana) were trained to search in a location defined by its geometric relationship to 2 landmarks. Two groups were trained to search at different points along the line connecting the landmarks, and 2 groups were trained to find the 3rd point of a triangle, on the basis of either direction or distance from the landmarks. All groups learned and transferred to new interlandmark distances. However, the constant-distance group learned more slowly, searched less accurately, and showed less transfer than the other 3 groups. When tested with new orientations of the landmarks, the birds tended to follow small but not large rotations. When tested with a single landmark, birds in the half, quarter, and constant-bearing groups searched in the appropriate direction from the landmark, but birds in the distance group did not. These results demonstrate that nutcrackers can learn a variety of geometric principles, that directional information may be weighted more heavily than distance information, and that the birds can use both absolute and relative information about spatial relationships.
\end{abstract}

Many experiments designed to address questions of spatial cognition use a hidden goal technique in which animals are trained to find a goal that bears a fixed relationship to a set of landmarks, which, in turn, have fixed geometric relationships to each other (e.g., Bennett, 1993; Bossema \& Pot, 1974; GouldBeierle \& Kamil, 1996, 1999; Spetch, Cheng, \& MacDonald, 1996; Spetch et al., 1997). The location of individual landmarks in the array is then manipulated to determine which geometric relationships are being used by the animal to find the goal (Cheng \& Spetch, 1998).

This is a powerful technique, but it has a disadvantage: The experimental manipulation of the landmark array changes landmark-landmark geometry. Therefore, when results indicate that animals are using geometric relationships with individual landmarks (rather than the geometric relationships among landmarks), it is under conditions in which both landmark-landmark and goal-landmark geometries have been altered. For example, when pigeons and humans were trained to find a goal in the center of a square array of four landmarks and then were tested with a rectangular array, pigeons (but not humans) searched off center in the rectangle, suggesting they were using goal-landmark relationships (Spetch et al., 1996, 1997). Changing the array from a square to a rectangle clearly alters landmark-landmark geometry. It is quite possible that the internal representations of the landmarks included information about landmark-landmark geometry but that this information was not expressed when elements in the landmark array were shifted. Similar results have been obtained with other alterations of landmark arrays (e.g., Spetch et al., 1996) and with other training arrays (e.g., Collett, Cartwright, \& Smith, 1986). However, this difficulty arises when animals are trained with an array that has fixed landmark-landmark and goal-landmark relationships and then tested with trans- formations of that array. In this context, note that the experiment that most clearly suggests learning of geometry in animals (Cheng, 1986) did not manipulate overall landmark configuration (see also Kelly, Spetch, \& Heth, 1998; Vallortigara, Zanforlin, \& Pasti, 1990).

Recently, Kamil and Jones (1997) attempted a new approach to the study of the use of geometric relationships by animals: systematically varying the relationship between a goal and landmarks so that the position of the goal was defined by a selected feature of the geometric relationship between landmarks. Clark's nutcrackers (Nucifraga columbiana) were trained to dig for a seed hidden halfway between two landmarks. Five interlandmark training distances were used, and the birds readily learned the task, generalizing to new distances interpolated between training distances. These experiments were quite different than several others that have presented evidence for learning to search a geometrically defined location such as the center of an arena (Tommasi, Vallortigara, \& Zanforlin, 1997) or partway between two walls (O'Keefe \& Burgess, 1996). The procedures we used clearly identified the specific landmarks on which the orientation was based, and the behavior of the nutcrackers was quite precise, centered on and within 8 to $10 \mathrm{~cm}$ of the correct location.

This combination of clear specification of the landmarks that control search and the precision of the search provided good evidence for the control of search behavior by some aspect of the geometry of the landmark array and its relationship to the goal location. The precise generalization to new interlandmark distances suggested that the nutcrackers had learned something that could reasonably be labeled a geometric rule, a method of solution based on distance and directional relationships that could be used with a variety of landmark configurations, particularly novel configurations. In the experi- 
ments reported here, we further investigated this issue in two ways: We tested to see if birds could extrapolate to distances outside of the range with which they were trained and to see if birds could learn problems in which the goal location was defined by geometric relationships other than halfway.

\section{Experiment 1}

\section{Method}

\section{Subjects}

Nineteen wild-caught adult Clark's nutcrackers with prior experience in landmark experiments (Gould-Beierle \& Kamil, 1996, 1998) were the subjects. Nine of the birds also had previous experience in operant experiments. One bird was dropped from the study because of difficulties during pretraining. The remaining 18 were assigned randomly to four groups (see below) with the constraint that previous experience was equalized across groups. Two of the birds were dropped from the study during habituation because of illness. Each bird was maintained at $90 \%( \pm 1 \mathrm{~g})$ of its free-feeding weight by controlled daily feeding of a diet consisting of turkey starter, sunflower seeds, parrot pellets, mealworms, pine seeds, and a vitamin supplement. The birds were housed individually with unlimited access to grit and water in a room maintained at $22^{\circ} \mathrm{C}$ with a 14:10-h light:dark cycle.

\section{Apparatus}

The room in which the experiment was conducted was 4.4 by $2.7 \mathrm{~m}$, with posters located on each of the north, west, and south walls. A porthole with a sliding door, which served as the entrance to the room for the birds, was located on the east wall just below a smoked glass observation window with a drawn curtain. The observer entrance door was south of the porthole on the east wall. Behind the porthole, outside the observation room, there was a holding cage from which the birds were released into the room and where the birds were kept between trials. A Panasonic videocamera (Model WV-BL200) was mounted in the ceiling above the test area and connected to a JVC BR3200 U video recorder and a Panasonic TR-930 video monitor located in the holding area. Subjects were observed through the observation window and on the video monitor. The test area was a wooden floor raised $7 \mathrm{~cm}$ above the concrete. It began $100 \mathrm{~cm}$ from the east door, covered the entire width of the room, and was covered with a $2-\mathrm{cm}$ layer of cellulose substrate. Four centrally located fluorescent lights illuminated the room. Two $40-\mathrm{cm}$ high and $2.54-\mathrm{cm}$ diameter pieces of polyvinyl chloride pipe, one green and one yellow, were used as landmarks.

\section{General Procedures}

Throughout all experiments, birds were carried by hand from their home cage to the holding cage outside the testing room. The lights in the holding room were off, and the lights in the testing room were on. A sliding door in the porthole was opened, and the bird was allowed to enter the room. When the bird was finished, the testing room lights were turned off, the sliding door was opened, the waiting room's lights were turned on, and the bird would fly back to the holding cage. After the completion of the daily trials, the bird was taken back to its home cage. Each trial continued until either the bird found the seed, the bird made 40 probes without finding the seed, or $10 \mathrm{~min}$ had passed. A probe was considered to have occurred whenever the beak came into contact with the substrate. All test sessions were videotaped.

\section{Habituation}

Habituation occurred for 2 days, with two trials per day. During the first day, there were four unshelled pine nuts in the room on each trial, two placed on each of two plastic $35-\mathrm{mm}$ film container lids placed on top of the substrate. During the second day, there were two seeds placed in the room for each trial. They were located one on top of the substrate and one buried about halfway beneath the substrate (with a lens cap beneath the second seed), to encourage the birds to probe the substrate to retrieve the second seed.

\section{Training}

Throughout training, the two landmarks were always present on each trial and were placed north (yellow) and south (green) of each other. The distance between the landmarks varied in increments of $20 \mathrm{~cm}$ from 38 to $98 \mathrm{~cm}$. The position of the landmarks determined the location of the pine seeds on each trial, but the rule relating landmark location to seed location was different for different groups.

The birds were randomly assigned to four groups that differed in how the target position was determined. (a) For birds in the half group, the target position was always located on the (imaginary) line that connected the two landmarks and halfway between them. (b) For birds in the quarter group, the target position was always located one fourth of the interlandmark distance from the yellow landmark (and three fourths of the interlandmark distance from the green landmark) on the line connecting the two landmarks. (c) For birds in the constantbearing group, the target position was always located at the third point of a triangle defined by the two landmarks. For these birds, the goal location was always at the intersection of two lines, which could be described in either absolute or relative terms. In absolute terms, one line described a bearing of northwest from the green landmark and the other a bearing of southwest from the yellow landmark. In relative terms, each line was at a $45^{\circ}$ angle between the hypotenuse and the line connecting the landmarks. The landmarks and the target position always described an isosceles right triangle. As the interlandmark distance varied, the bearings from the landmarks to the target were constant while the distances from the landmarks to the target were variable. (d) For birds in this constant-distance group, the target position was also located at the third point of a triangle. In this case, the third point was located at the intersection to the west of the landmarks of two circles, each of 55-cm diameter, one centered on each landmark. Thus for the constant-distance group, as the interlandmark distance varied, the bearings from the landmarks to the target varied, but the distance between each landmark and the goal remained constant. The conditions for the constant-distance and constant-bearings group were chosen so that when the interlandmark distance was $78 \mathrm{~cm}$, both groups were being tested with the same triangle.

To ensure that only the relationship between the landmarks and the target position could be used to find the buried seed, the position of the landmarks was varied across trials. Given the size of the room and the interlandmark distances used, the total area in which the array could be placed varied by $120 \mathrm{~cm}$ in the east-west (EW) direction and $80 \mathrm{~cm}$ in the north-south (NS) direction. In addition, we did not allow any landmark to be placed within $35 \mathrm{~cm}$ of a wall or the east edge of the test area. We designated 116 possible locations for the target location (in a $12 \times 8$ grid), chose 1 at random for each trial (without replacement until all had been used and with the restriction that the positions be located in each of the four quadrants of the room each day), then allowed that location to determine the position of the landmarks for that trial.

During each day of training, each bird received four trials, one at each of the four interlandmark distances, in random order. For the first 30 days, the seeds were partially exposed above the substrate during the first three trials and were completely buried during the last trial, the test trial. For the next 11 days, the seed was completely buried on the last two trials. For the next 13 days, the seed was buried on all four daily trials, and on the last day, only two trials were conducted, with buried seeds on both, making 100 buried-seed trials in total.

\section{Data Recording, Reduction, and Analysis}

All buried-seed test trials were videotaped. The videotape for each session was played back on an NEC JC-1401 computer monitor connected to a TARGA videographics board and a Panasonic AG-1730 
VCR that allowed slow-motion playback. When a bird made a probe, the videotape was paused, and the location of the probe was marked with an observer-controlled pad system that controlled a cursor on the monitor. Each probe was then automatically assigned $X$ and $Y$ coordinates, which were used to calculate three values for each probe: (a) $\mathrm{Er}$ ror distance - the absolute value of the distance between each dig and the correct location was averaged separately in the NS and EW axes, and then the Pythagorean theorem was applied to these distances to calculate the error distance. (b) NS axis error - the distance in centimeters from the correct location along the NS axis (parallel to the imaginary line connecting the landmarks) was calculated. (c) EW axis errorthe distance in centimeters from the correct location along the EW axis (perpendicular to the line connecting the landmarks) was calculated.

These error distances were calculated separately for each probe and were assigned an absolute value. That is, averaging was not allowed to affect the accuracy estimates. If a bird probed $3 \mathrm{~cm}$ to the west then $3 \mathrm{~cm}$ to the east of the target position, its mean error score for the two probes would be 3 , not 0 . During acquisition, either all probes until the goal was uncovered or the first five probes of each test session, whichever came first, were included in analysis. Note that this measure has several advantages over measures that calculate only a central tendency for search location, such as the iterated procedure that locates peak search (Cheng, 1989). Our measure provides evidence about both the mean location of search relative to the goal and the precision of that search.

Analyses of variance (ANOVAs) were then used to analyze the data. We carried out subsequent tests, either Fisher's least significant difference (LSD) test $(\alpha=.05)$ or focused ANOVAs, only after significant overall $F$ ratios. In most cases, we first analyzed total error, to compare general accuracy of the groups. We followed this overall analysis with more detailed analyses, looking at error as a function of EW and NS axes (axis was a factor in these ANOVAs). These analyses, although quite different, are not completely independent from the analysis of total error. Therefore, we report all significant $F$ ratios from the total-error ANOVAs but do not report redundant $F$ ratios from the second set.

\section{Results}

Acquisition was analyzed by dividing the 100 buried-seed acquisition trials into ten 10 -trial blocks. Total error distance was analyzed with a Groups $\times$ Blocks mixed ANOVA. There was a significant decline in error as acquisition proceeded, $F(9,126)=$ $5.50, p<.001$, as well as significant differences among groups, $F(3,14)=5.01, p<.02$. A subsequent Fisher's LSD test showed that the quarter group was significantly better than the half and constant-distance groups and that the quarter, half, and constant-bearing groups performed significantly better then the constant-distance group (see Figure 1). Then, to analyze possible differences in performance between the NS and EW axes, we performed a second ANOVA with group, block, and axis as independent variables. Performance was significantly better in the EW than in the NS axis, $F(1,14)=10.06, p<.01$, and there was a significant Group $\times$ Axis interaction, $F(3,14)$ $=6.76, p<.01$ (see Figure 2). With follow-up ANOVAs, we found that the difference between axes was significant for the half group, $F(1,4)=27.58, p<.01$, but not for any other group $(p>.125$ in all cases). Maps showing the location of search at the end of training are shown in Figure 3.

Overall search accuracy at the end of acquisition was analyzed with the data from the last three blocks. Because these trials provided relatively few pieces of data per bird per condition, we eliminated as outliers any trials during which total error was greater than $70 \mathrm{~cm}$ (less than $1 \%$ of the total). A Groups $\times$ Interlandmark Distance ANOVA indicated there were significant differences among the groups, $F(3,14)=6.55$, $p<.01$. Subsequent Fisher's tests showed that the quarter group performed more accurately than the half and distance groups and that the bearing group was more accurate than the distance group.

Our landmark arrays were arranged so that all groups were tested with the same set of interlandmark distances. Therefore, they experienced different goal-landmark distances. When accuracy for each group was examined as a function of goallandmark distance (see Figure 4), there was a general trend toward increased accuracy at shorter goal-landmark distances, as would be expected from the significant effects of landmarklandmark distance in previous analyses. The correlation be-

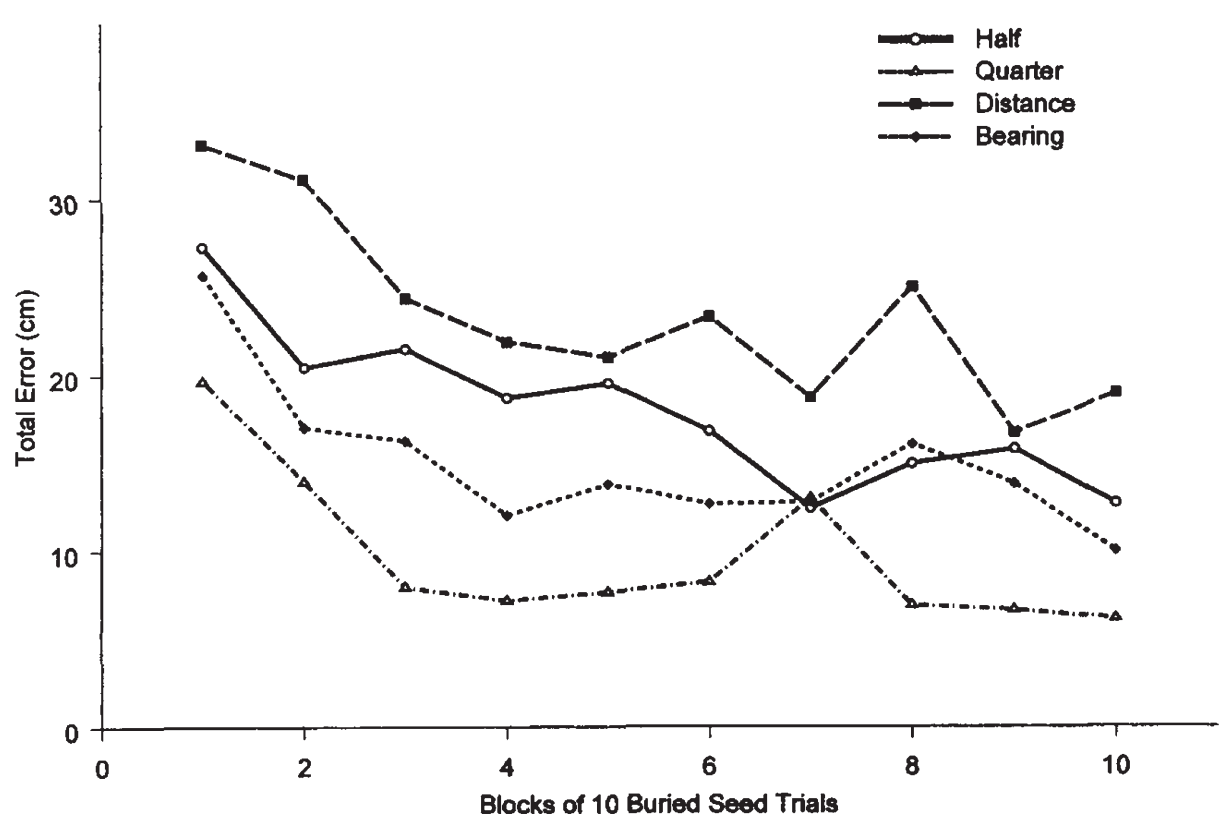

Figure 1. Mean total error for each group for each block of 10 buried-seed trials during Experiment 1. 

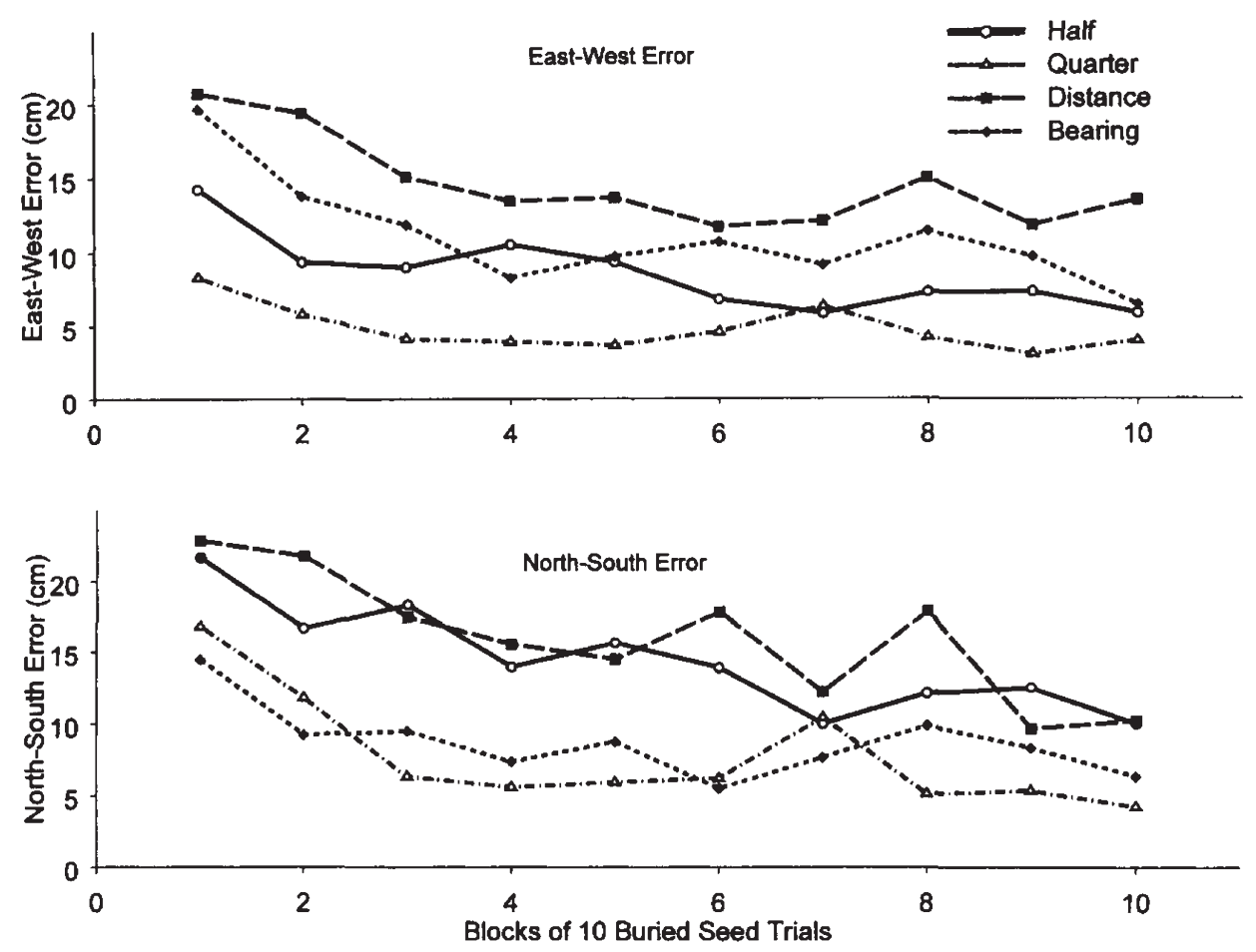

Figure 2. Mean error in the east-west (top panel) and north-south (bottom panel) axes during each 10-trial block of Experiment 1.

tween goal-landmark distance and search accuracy (across all groups) was .57, $p<.01$. We also examined the linear and quadratic components of the effects of goal-landmark distance on search accuracy separately for the quarter, half, and bearing groups (the distance group was always tested at the same goal-landmark distance). We found that the only significant effect was a linear trend in the case of the bearings group ( $p$ $<.01$ ). Finally, we compared the accuracies of the bearing and distance groups in two ways. First, we included all trials during which goal-landmark distance was $55 \mathrm{~cm}$ (all trials for the

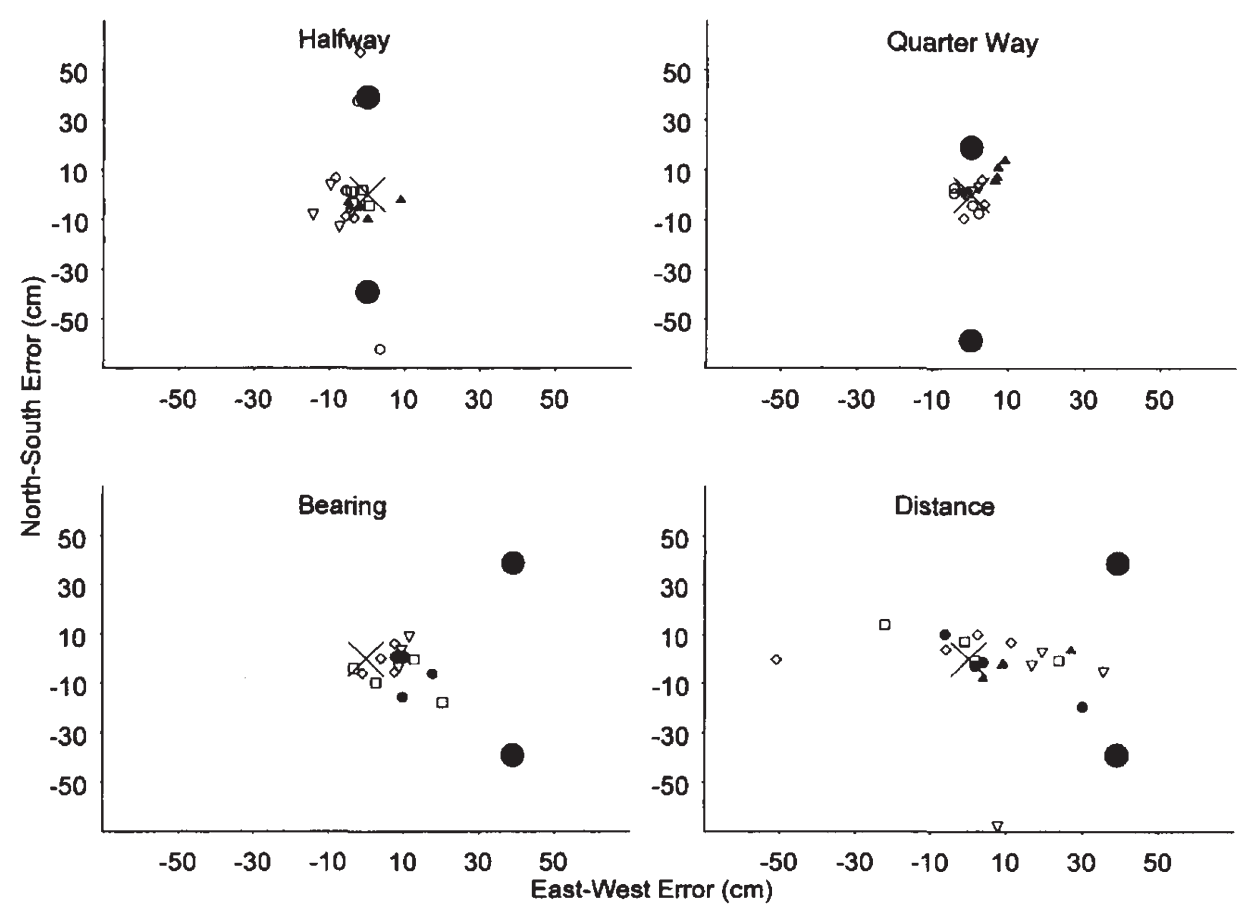

Figure 3. Maps showing the location of search for each bird in each group during each of the last four sessions with an interlandmark distance of $78 \mathrm{~cm}$ during Experiment 1 . Each point represents a single session, and each symbol represents an individual bird. The large circles show the location of the landmarks, and the $\mathrm{X}$ shows the correct location for that group. 


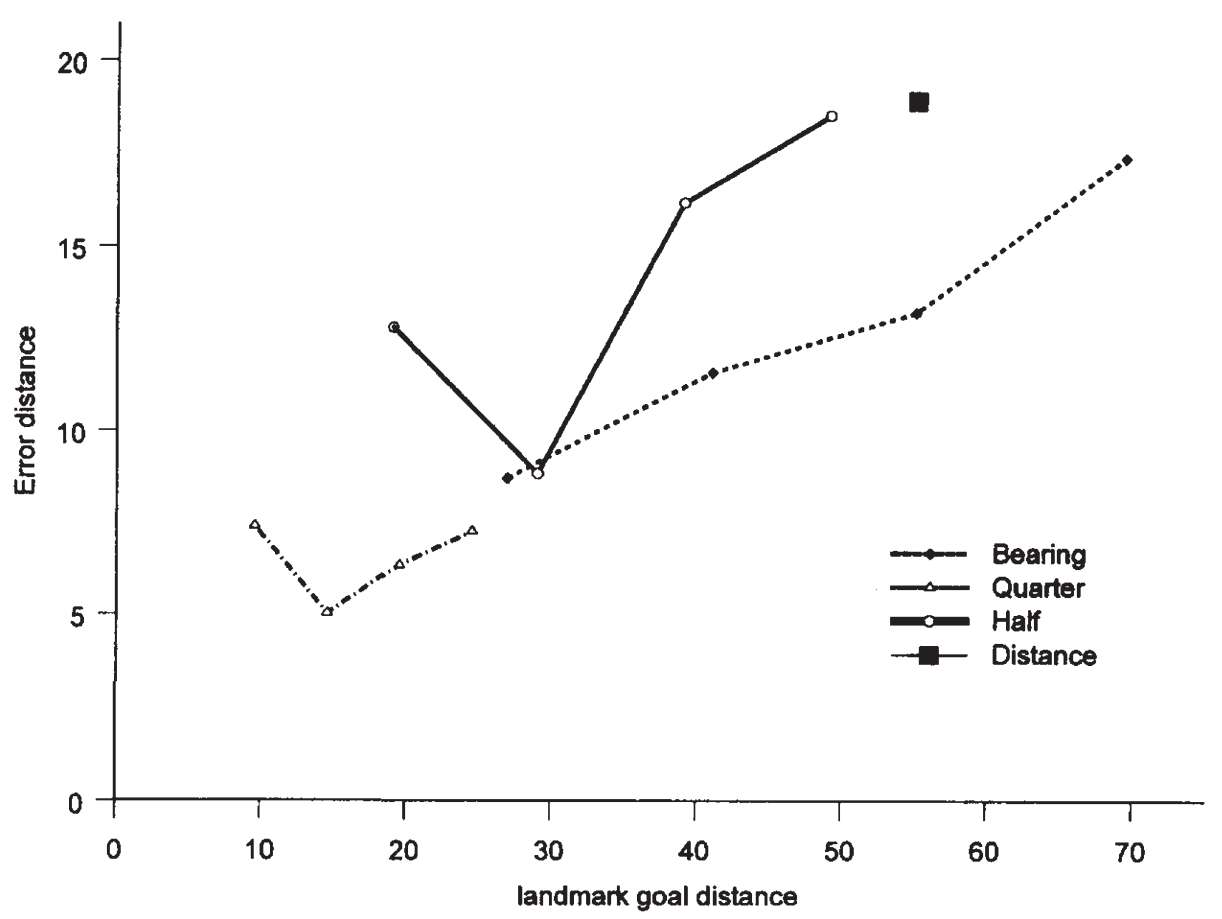

Figure 4. Mean error for each group as a function of the distance between the goal and the landmarks. In the case of the quarter group, the distance to the closest landmark was used. The distance group is represented by a single point because the goal-landmark distance was held constant for these birds

distance group and 78-cm interlandmark distance for the bearing group). The bearing group tended to perform more accurately than the distance group, but the difference was not significant, $t(7)=2.12, p<.10$. In a second analysis, we used only data from trials during which the birds in both groups were presented with identical landmark arrays $(78-\mathrm{cm}$ interlandmark distance), goal-landmark distance $(55 \mathrm{~cm})$, and goal location. In this case, the bearing group tended to be more accurate than the distance group, but the difference was not significant, $t(7)=2.07, p<.10$.

\section{Discussion}

These data replicate and extend the findings of Kamil and Jones (1997) in several important ways. They demonstrate that Clark's nutcrackers can learn to solve problems whose solution depends on the geometric relationships among landmarks and a goal and that this ability is not limited to bisection. The learning by the quarter group shows that nutcrackers can learn to find positions on the line connecting the landmarks other than halfway and implies that they could learn any proportional distance. The learning by the constant-bearing and constant-distance groups demonstrates that geometric relationships that define points off the connecting line can also be learned. The birds were able to solve problems in which the underlying principle was primarily directional (constant bearing) and in which it was primarily distance based (constant distance).

The data also suggest that some geometric relationships are learned more easily than others. The quarter group consistently performed better than the other groups, including the half group, and the distance group generally had lower accuracy than the other groups, including the bearing group. These group effects, especially in the rate of acquisition, could reflect differences in how well the different relationships were learned.
However, these group differences, especially at the end of acquisition, could also be the result of differences in the difficulty of locating the correct location specified by the different geometric relationships required of each group. This is particularly true because the different geometric principles used led to different goal-landmark distances in many cases and several studies have demonstrated that landmarks close to a goal exercise greater control over search than landmarks further from the goal (e.g., Bennett, 1993; Cheng, 1989). It seems most likely that this alternative explanation accounts for the performance of the quarter group, because the goal location for that group was particularly close to a landmark. However, this seems less likely to account for the poor performance of the distance group. The goal-landmark distances for this group were only somewhat longer, on average, than those for bearing group. In addition, the performance of the distance group, which always had a goal-landmark distance of $55 \mathrm{~cm}$, tended to be less accurate than the performance of the bearing group when their goallandmark distance was $55 \mathrm{~cm}$. This suggests a qualitative difference between what the distance and bearing groups learned, although further data on this issue are needed.

Biegler, McGregor, and Healy (1998; see also Kamil \& Jones, 1998) have suggested that nutcrackers may solve geometric problems such as halfway between two landmarks by learning separate landmark-goal vectors for each interlandmark distance used during training. For example, birds in the half group might have learned a conditional discrimination based on the interlandmark distance, of the form "if the landmarks are $X \mathrm{~cm}$ apart, search $Y \mathrm{~cm}$ southeast of the northern one." Such conditional learning would not directly or indirectly involve the constant relationship that $Y$ is one half of $X$. However, the relatively large differences between the bearing and distance groups suggest that separate learning for each interlandmark distance is unlikely. If the vectors defining the goal 
location are learned separately for each interlandmark distance, there is no reason for the bearings group to learn more rapidly and perform more accurately than the distance group. It is possible that the distance group did learn four separate problems while the bearing group learned some general principle. Further tests, such as transfer tests with new interlandmark distances, are necessary to resolve this issue.

Kamil and Jones (1997) have found that error in the EW axis is smaller than error in the NS axis, especially at longer interlandmark distances. When the goal location is between a pair of landmarks, search involves two components: the directional problem of locating the line connecting the landmarks and the distance-estimation problem of locating the correct location along that line. Because the landmarks were located north and south of each other, these results suggest that the distance judgment required along the NS axis is more difficult than the directional judgment required along the EW axis. In the current experiment, the same difference was found during acquisition, but only for the half group. This is not inconsistent with our original interpretation. For the quarter group, the goal was close to one landmark, which probably reduced the difficulty of this distance judgment (as noted above). For the bearing and distance groups, the goal location changed in both axes as interlandmark distance changed. Thus for these two groups, the judgments that needed to be made were similar for the two axes.

The comparison of the performance of the different groups along the NS axis with performance along the EW axis offers some indications of the decision processes that the nutcrackers used to find the correct location of the buried seed. For the quarter and half groups, logic suggests that there are two qualitatively different decisions involved in finding the correct position: locating the line connecting the two landmarks and then finding the correct spot along that line. One decision (in the EW axis) involves bearings, whereas the other (in the NS axis) involves distance. The finding of greater accuracy in the EW then in the NS axis for the half group suggests that directional information can lead to more accurate performance then distance information. This is consistent with the poorer performance of the constant-distance group than the constant-bearing group during acquisition and transfer testing.

The difference between the axes was not significant for the bearing and distance groups. But the EW versus NS distinction is less meaningful for these groups than for the half and quarter groups. This is because the correct location for the bearing and distance groups changed in both axes as interlandmark distance changed. Thus, for these two groups, the nature of the decision to be made did not vary as a function of axis. The decision was entirely directional in each axis for the bearing group and entirely distance-based for the distance group.

\section{Experiment 2}

Kamil and Jones (1997) found that birds trained to dig halfway between landmarks that were 20, 40, 60, 80, 100 and 120 $\mathrm{cm}$ apart generalized virtually perfectly to new interlandmark distances of 30, 50, 70, 90 and $110 \mathrm{~cm}$. Although that finding and the data of Experiment 1 are consistent with the idea that the nutcrackers learned to find the goal using the same principle or rule for all landmark arrays, it is possible that in the current experiment at least some of the groups did not learn a general principle but rather learned 4 separate problems defined by the four different interlandmark distances to which they were exposed during acquisition. Biegler et al. (1998) have pointed out that this kind of conditional learning could produce good performance at new interlandmark distances that were within the training range (interpolation). However, birds that had learned such a conditional discrimination would not be able to generalize accurately to interlandmark distances outside the training range (extrapolation; Biegler et al., 1998). Experiment 2 was designed to test the birds ability to deal with new interlandmark distances, both within and beyond the training range.

\section{Method}

\section{Procedure}

The nutcrackers of Experiment 1 served in Experiment 2, which began immediately after Experiment 1 ended. All procedures were identical to those of Experiment 1 except for the values and scheduling of interlandmark distances and the introduction of no-seed trials.

\section{Introduction of No-Seed Test Trials}

During the first stage of Experiment 2, which lasted 8 days, the nutcrackers were introduced to occasional probe trials during which no seeds were present. The purpose of this stage was to introduce the noseed procedure for use during probe trial tests. Several studies have demonstrated that nutcrackers do not use odor to locate buried seeds (e.g., Balda, 1980; Kamil, Balda, Olson, \& Good, 1993; Kamil \& Jones, 1997). However, no-seed probe trials presented as part of a series of trials in which there are also control trials without seeds minimize any learning that might take place during the probe trials.

All procedures were identical to testing at the end of Experiment 1 except that during one of each day's four test trials, no seed was present. The no-seed trial was randomly assigned to either the second, third, or fourth trial. No-seed trials continued until the nutcracker made five to seven probes at the substrate (five was the criterial number, but some birds probed so rapidly that they sometimes managed six or seven probes before the lights could be turned off). Each of the four interlandmark distances was tested equally often with the noseed procedure.

\section{Transfer Testing}

Each day for the next 27 days, the birds received four trials, including one no-seed test trial. The no-seed test trial was randomly assigned within the day with the constraint that it never occurred on the first trial. On trials with seeds, only the original training distances were used, in random order with the restriction that no single interlandmark distance was used more than once per day. On the no-seed test trials, nine interlandmark distances were used, from 28 to $108 \mathrm{~cm}$, in 10-cm steps. This series included the original training distances as well as two new distances outside of the training range (extrapolated distances, 28 and $108 \mathrm{~cm}$ ) and three new distances within the training range (interpolated distances, 48,68 , and $88 \mathrm{~cm}$ ). Across the 27 days of transfer testing, each bird was tested three times on no-seed trials at each of the nine interlandmark distances, in a randomized block design. Only the data from the first five digs of the no-seed trials were used in data analysis.

\section{Results}

We first compared performance on no-seed trials with performance on trials with a seed buried at the goal. We included only the first two probes from each trial because the birds usually found the seed within two to three probes on seed trials and using more probes would introduce a bias. For example, 


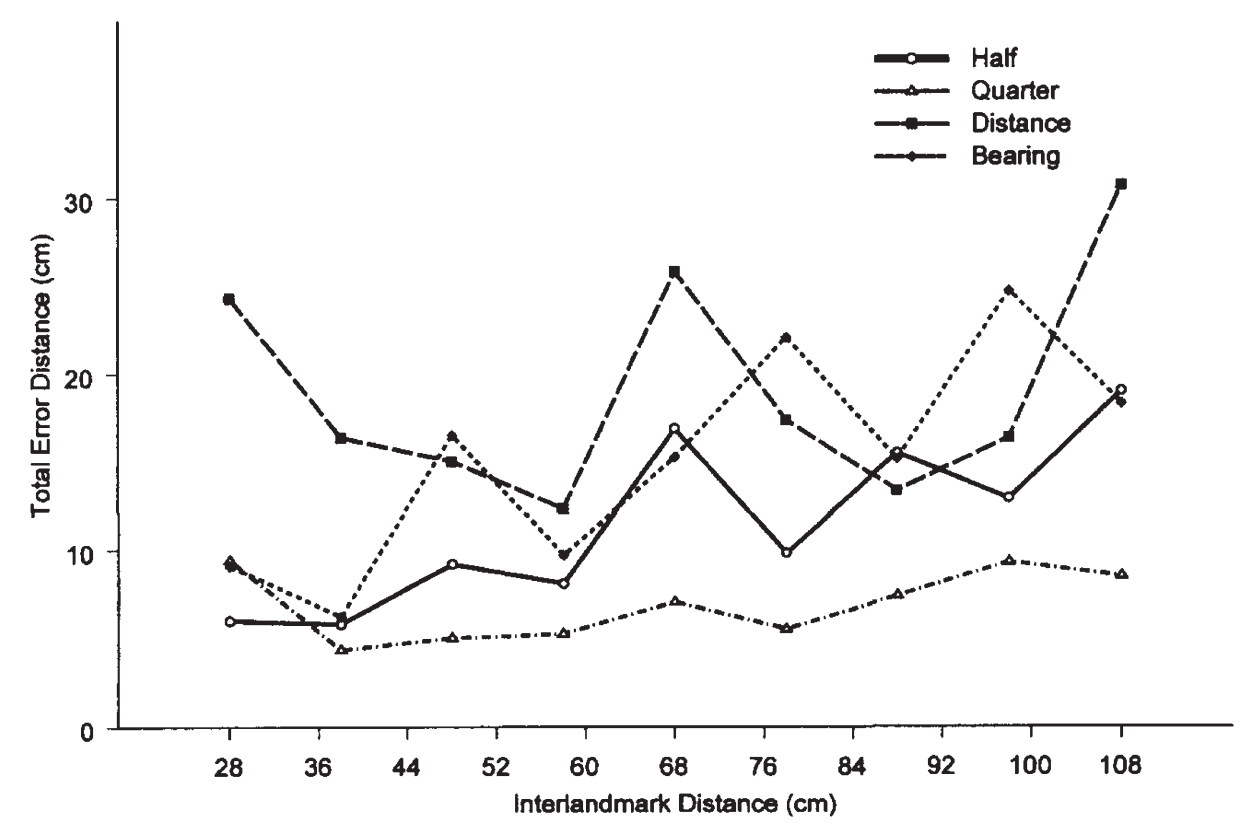

Figure 5. Mean error for each group at each interlandmark distance during the transfer test of Experiment 2. Distances used during training were 38, 58, 78, and $98 \mathrm{~cm}$. Interpolated distances were 48, 68, and $88 \mathrm{~cm}$. Extrapolated distances were 28 and $108 \mathrm{~cm}$.

imagine that a bird made five digs on a no-seed trial, producing errors of 4, 0, 4, 10, and $7 \mathrm{~cm}$, in that order, giving an average error score of $5 \mathrm{~cm}$ per dig. Search in the same locations during a trial with a seed would yield an average error of $2 \mathrm{~cm}$ per dig, because the trial would end with discovery of the seed on the second dig and the last three digs would never occur. To determine if the absence of a seed affected performance, we subjected total error distance data, collected during the introduction of no-seed trials period, to an ANOVA in which seed or no seed and groups were the factors. The mean error was $13.15 \mathrm{~cm}(S D=0.70)$ for trials with seeds and $14.9 \mathrm{~cm}(S D=$ 1.25) for trials without seeds. This difference was not significant, $F(1,14)=2.39, p>.10$ ), nor was the Seed (present vs. absent) $\times$ Group interaction $F(3,14)=1.08, p>.25$.

We first analyzed the results of transfer testing on total error distance with a two-way mixed ANOVA, with group and interlandmark distance as factors. There were significant differences among the groups, $F(3,14)=12.00, p<.001$, showing the same pattern obtained during Experiment 1 (see Figure 5). There was also a significant increase in error distance as interlandmark distance increased, $F(8,24)=4.23, p<.001$, and a significant Group $\times$ Interlandmark Distance interaction, $F(24$, $112)=2.72, p<.05$. Exploring the nature of this interaction was somewhat complicated because there was a general increase in error as interlandmark distance increased and because the transfer distances were thoroughly interspersed with training distances. Therefore, we explored the nature of this interaction by conducting separate ANOVAs for each group followed by a set of Fisher's tests for the effects of interlandmark distance. The Fisher's tests were selected so that as a set they tested whether error distance differed between any interlandmark distance and its immediate neighbors (e.g., 28 and 38 or 38 and 48) because every pair in these comparisons involved a training and a transfer distance. There were 32 such compari- sons in total, 8 for each group. Only 2 were significant. For the quarter group, error was significantly lower at an interlandmark distance of $58 \mathrm{~cm}$ than $68 \mathrm{~cm}$ (Fisher's exact test, $p<.05$ ). For the distance group, error distance was greater at $108 \mathrm{~cm}$ than at $98 \mathrm{~cm}$ (Fisher's exact test, $p<.05$ ). In addition, because the comparison of performance at the 28 - and $38-\mathrm{cm}$ interlandmark distances was of particular theoretical importance (see below), we also carried out separate Group $\times$ Group $t$ tests for differences between these two interlandmark distances. None were significant: Quarter, $t(3)=1.12, p>.30$; half, $t(4)=0.32, p$ $>.50$; bearing, $t(4)=0.92, p>.40$; distance, $t(4)=1.46, p>.20$.

To examine behavior during the transfer tests more closely, we calculated the distributions of digging along each axis for each group (see Figure 6). Because interlandmark distance varied, each bin was defined as a constant proportion of interlandmark distance. In almost all cases, the distribution of digging on trials with the training interlandmark distances were very similar to those on trials with either interpolated or extrapolated interlandmark distances. The clear exceptions occurred when the constant-distance group was tested with the extrapolated interlandmark distances. During extrapolated test trials, the EW distribution was virtually flat, with no peak located near the point $55 \mathrm{~cm}$ from each landmark. In the case of the NS distribution, there was a peak in the correct place on extrapolated trials, but it was much less pronounced than on other test trials.

To analyze this aspect of performance, we determined the interquartile range (in centimeters) for each bird and condition. These scores were then analyzed with a Group $\times$ Interlandmark Condition ANOVA, where there were three conditions: training $(38,58,78$, and $98 \mathrm{~cm})$, interpolated $(48,68$, and $88 \mathrm{~cm}$ ) and extrapolated $(28$ and $108 \mathrm{~cm})$. There were no significant effects in the EW axis. However, in the NS axis, there was a significant groups effect, $F(3,14)=20.75, p<.001$, a sig- 

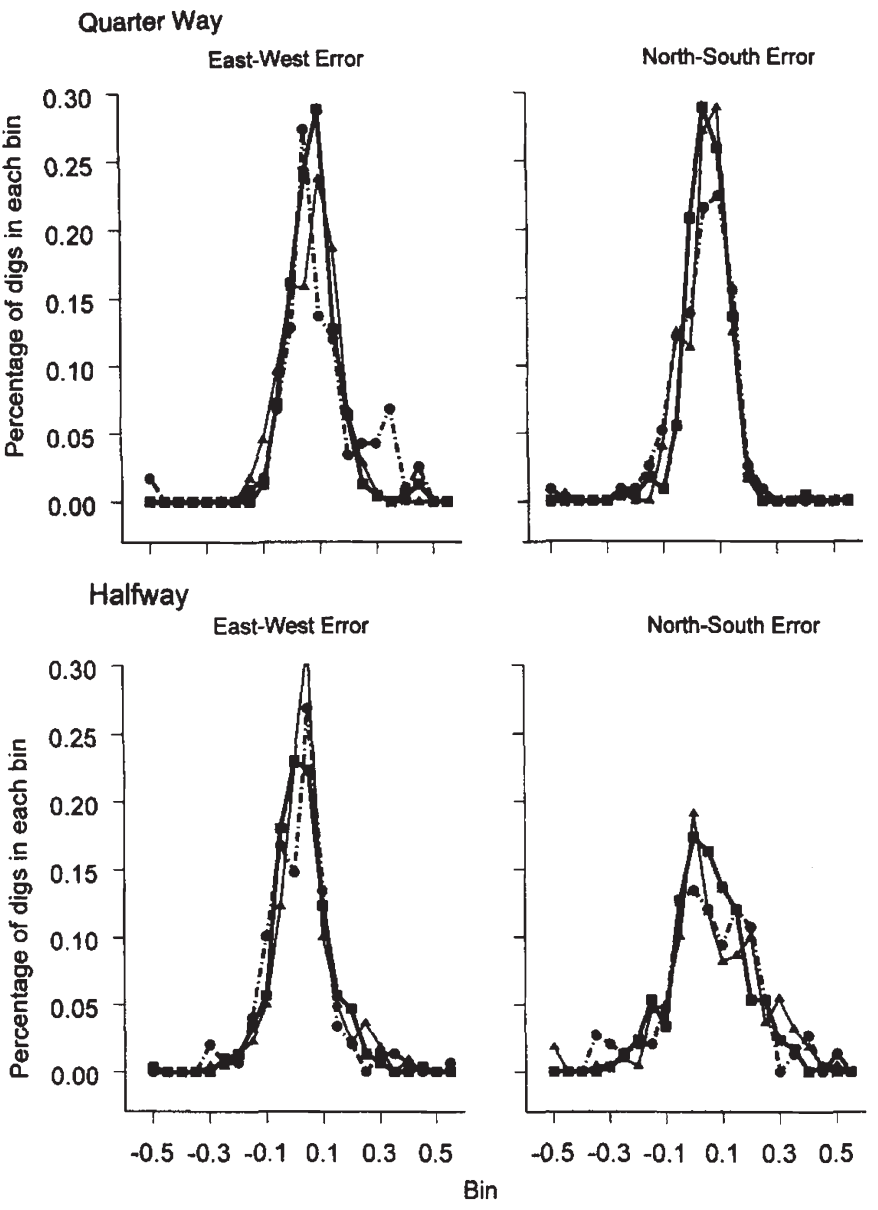

\section{Constant Bearing}

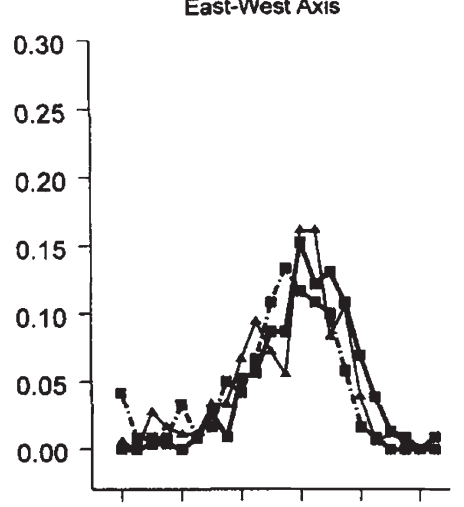

Constant Distance

East-West Error

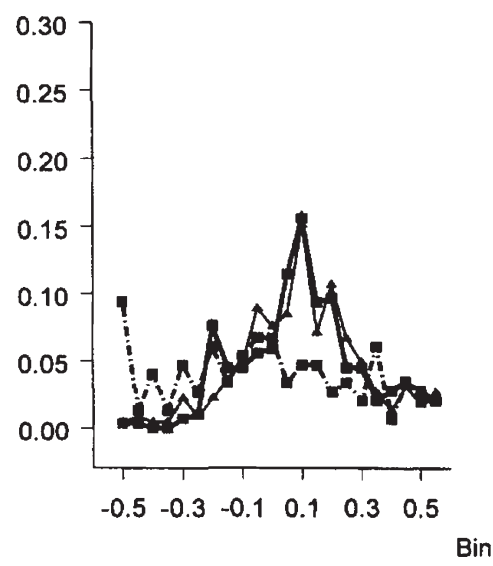

North-South Axis
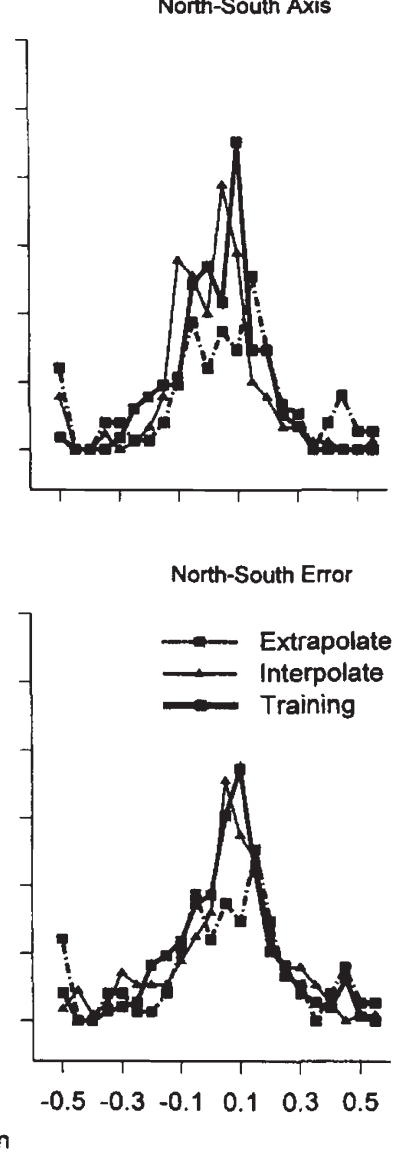

Figure 6. The distribution of search for each group during transfer tests. Each of the first five probes from each session is included. The distributions were divided into bins (shown along the x-axis) in which the error in centimeters was divided by the interlandmark distance, allowing all errors to be included on a common axis. The distributions of error in the north-south axis and error in the east-west axis are shown.

nificant condition effect, $F(2,28)=4.54, p<.05$, and a significant interaction, $F(6,28)=2.63, p<.05$. The nature of the Group $\times$ Condition interaction was explored by conducting separate ANOVAs of the effects of condition for each group. There were no significant effects of interlandmark condition for the quarter, half, or bearings groups $(p>.25$ in all cases); however, there was a significant effect for the distance group, $F(2,8)=5.89, p<.05$. A subsequent Fisher's test found that this was due to greater error in extrapolation testing than either interpolation or training-distance testing.

\section{Discussion}

The most important results of the transfer tests of Experiment 2 can be summarized quite simply: Generalization to new interlandmark distances was always very good except when the distance group was tested with interlandmark distances outside of the range used during training. With that one exception, transfer was apparent in two ways. Search on probe trials with new interlandmark distances was located very near the location of search on trials with training distances, as indicated by the modes of the search distributions. Second, the precision of search was unaffected, as reflected in the amount of varia- tion in the search distributions. The ability of birds in the half, quarter, and constant-bearings groups to perform well at extrapolated as well as interpolated distances gives added weight to the argument that the basis on which the birds learned to solve these tasks was general. It is reasonable to conclude that the birds in the quarter, half, and bearings group learned some single rule applicable to many arrangements of the landmarks rather than treating each landmark array presented during acquisition as a separate problem. Principles based on aspects of the geometry of the locations of goals and landmark arrays can be generalized, and this generalization can occur with extrapolated as well as interpolated interlandmark distances.

Although the generalization to new interlandmark distances demonstrates that the birds in the quarter, half, and distance groups learned some sort of rule, the data do not establish either precisely which rules were used or the structure of those rules. There are many possibilities. For example, the simplest explanation for the performance of the bearing group may be that they learned to search at the intersection of two bearings, one northwest from the green landmark and the other southwest from the yellow landmark. However, there are many other possibilities. For example, the birds also could have learned to search along the line composed of locations 


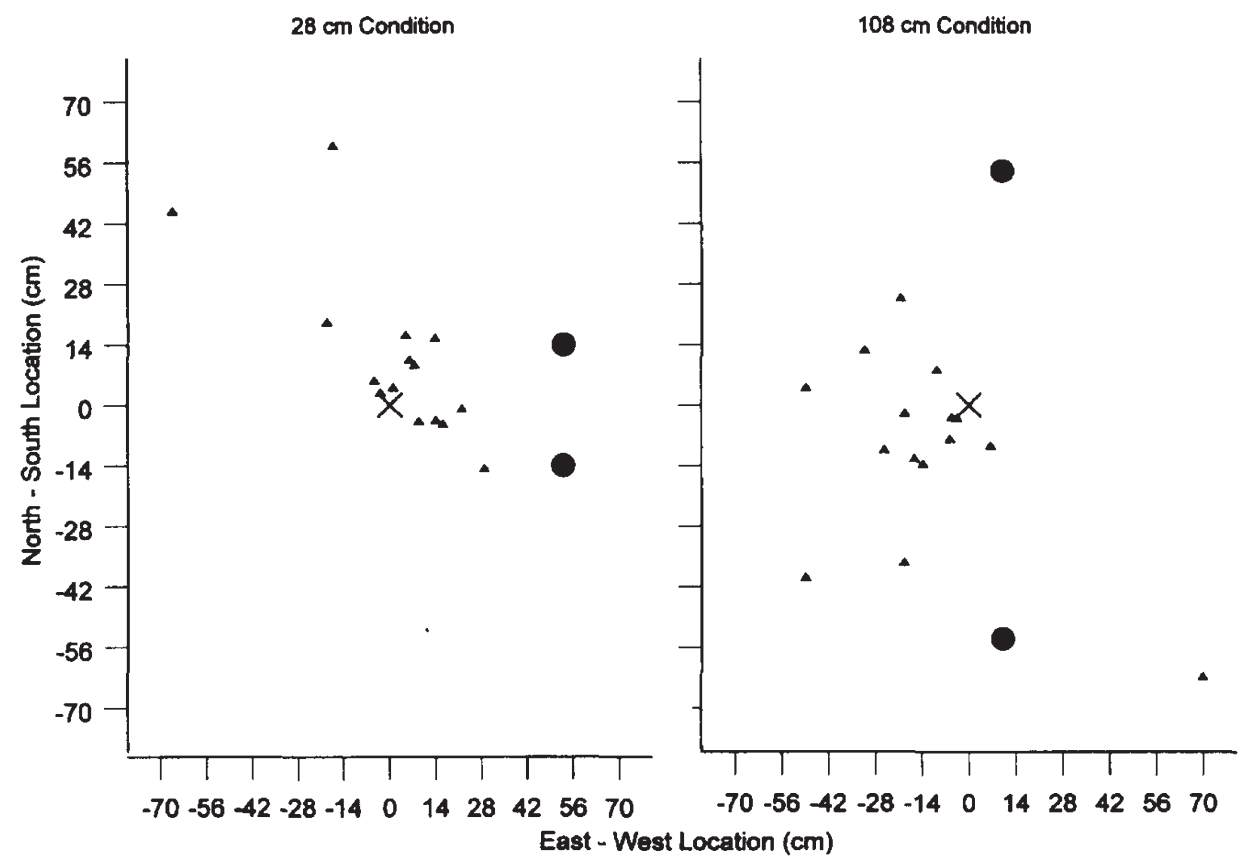

Figure 7. Maps showing the location of digging for each bird in the constant-distance group during probe testing at the extrapolated distances of 28 and $108 \mathrm{~cm}$. Each point represents the mean location of the first five digs of a single session. The large circles show the location of the landmarks, and the $\mathrm{X}$ shows the correct location

equidistant from the landmarks at the point at which the relative angular distance (or relative bearing) between the landmarks was $90^{\circ}$. Furthermore, specification of a particular rule will require experiments to determine the stimuli that the animal uses to carry out that rule. For example, there are many different "compasses" that can be used by animals to determine a bearing (reviewed by Gallistel, 1990). Further work will be needed to delineate and test the nature of the rules learned by the birds when faced with problems during which goal location is defined by abstract geometrical relationships.

The failure of the distance group to generalize on extrapolation probe trials is apparent in terms of both the precision and modal location of their search. There was no single mode in the EW axis, and the distribution of search was very broadly distributed in both axes, especially EW. The relatively better performance in the NS axis by these birds indicates that they tended to stay on the line that runs perpendicular to and equidistant from the two landmarks. The poor performance in the EW axis indicates that they searched fairly indiscriminately along the equidistance line. Visual inspection of the maps showing the location of digs during testing (see Figure 7) clarifies what happened, suggesting that the distance group may have learned interlandmark-specific rules. When the landmarks were presented at the smallest interlandmark distance $(28 \mathrm{~cm})$, the height of the resulting triangle was greater than that of any of the triangles that held during training, and the nutcrackers tended to stay too close to the line connecting the landmarks. The opposite was true at the largest interlandmark distance.

As pointed out in the discussion of Experiment 1, the relatively poor performance of the distance group compared with that of the other three groups could have resulted from either differences in how well the relevant geometric relationships were learned or differences in the difficulty of locating the correct location specified by the different geometric relationships required of each group. The failure of the distance group to generalize to extrapolated distances suggests that the constantdistance problem is more difficult to learn than the others, not just more difficult in terms of its psychophysics. It seems likely that the basis on which the tasks were learned was different for the constant-distance birds than for the birds in the other three groups. It would be of interest to train several distance groups, each with a different critical goal-landmark distance, and observe the effects on acquisition and transfer.

Biegler et al. (1998) have suggested that the birds in the Kamil and Jones (1997) experiment learned a set of independent problems, one for each interlandmark distance. They have pointed out that such learning can provide a basis for accurate performance at interpolated distances, but not at extrapolated distances, exactly the pattern observed with the distance group. The most tenable hypothesis for the differences between the groups is that the distance group learned four separate problems, as suggested by the model of Biegler et al., whereas the other three groups learned a single problem.

\section{Experiment 3}

There are different ways animals can use landmark-based bearings while searching or navigating. Consider, for example, a bird that has learned to search on the line between two landmarks that are north and south of each other. One way to identify the line is through an absolute bearing (e.g., north of the south landmark). In this case, changing the orientation of the landmarks by rotating the array will result in search that is no longer on the line. Another way would be to use relative bearings based on the configuration of the landmark array, in 
which case search should remain on the line after rotation of the array. When Kamil and Jones (1997) tested the effects of array rotation, they found that the birds tended to stay on or near the line after moderate rotations of the landmark array, but not when the landmarks were rotated $90^{\circ}$ or $180^{\circ}$. During this experiment, we explored the effects of small and large changes in the NS orientation of the landmarks and of the removal of one of the landmarks.

\section{Method}

Conditions were generally the same as during Experiment 2. Each bird received four trials a day, one at each of the training interlandmark distances $(38,58,78$, and $98 \mathrm{~cm})$. One of the last three trials each day (randomly determined) was a no-seed trial and could be either a test or a control trial. The order of test and control trials was randomized separately for each nutcracker.

During the first 24 days of Experiment 3, we tested small rotations. The landmarks were presented in one of three orientations on the noseed trial each day: the normal, NS orientation or rotated $22^{\circ}$ clockwise or counterclockwise. All rotations during Experiment 3 were carried out with the midpoint, between the landmarks as the origin. Each condition was presented once in each set of 3 consecutive days, in random order within the days. Across the entire experiment, each of the three orientations was tested twice at each of the four interlandmark distances.

During the next 14 days, we tested the effects of large changes in the orientation of the landmarks. The landmarks were presented in one of four orientations on the no-seed trial each day: control orientation, $90^{\circ}$ clockwise rotation, $90^{\circ}$ counterclockwise rotation, and $180^{\circ}$ rotation. All of these manipulations were carried out with an interlandmark distance of $78 \mathrm{~cm}$. Randomization and counterbalancing were as during the previous stage.

During the last 8 days, we tested the behavior of the birds when only a single landmark was presented. Three conditions were presented during the daily no-seed trial: control (four times), yellow landmark only (two times) and green landmark only (two times). Tests and control were presented on alternating days.

\section{Results}

The effects of rotation on the location of the correct (rotated) point were different for different treatments as well as different groups. Therefore, different analyses were often required for the effects of each treatment and each group.

\section{Small Rotation}

Because all groups were tested with the small rotation at four interlandmark distances, the error data were first analyzed with a Groups $\times$ Interlandmark Distance $\times$ Rotation (rotated vs. control, collapsing across clockwise and counterclockwise rotations) ANOVA. As during acquisition and transfer testing, there were significant effects for group, $F(3,14)=15.79, p<$ .001 , interlandmark distance, $F(3,42)=7.50, p<.001$, and rotation, $F(1,14)=12.65, p<.01$. Mean error was $14.2 \mathrm{~cm}(S D=$ 1.11) under the control condition and $17.6 \mathrm{~cm}(S D=0.77)$ when the landmarks were rotated (see Figure 8). There were no significant interactions.

In the light of this relatively small effect of the $22^{\circ}$ rotation, we conducted more detailed analyses for each group. In these analyses, we calculated the location of the lines that would be described by both absolute and relative bearings and the mean position of the first five probes of each trial. We then calculated the distance from the mean search location to each of these lines. The different goal-landmark geometries required different procedural details for some groups than for others.

Half and quarter groups. The distance between mean search location and both the line connecting the rotated landmarks and the NS lines from the individual landmarks was calculated. We then compared the distance to the rotated line connecting the landmarks with the distance to whichever NS line came closest to the dig position with a Line Type $\times$ Interlandmark Distance repeated measures ANOVA. The distances to the two different line types (relative vs. absolute bearings) did not differ significantly, averaging $5.65 \mathrm{~cm}(S E=0.88)$ and $6.76 \mathrm{~cm}(S E=0.89)$, respectively. Both distances increased with interlandmark distance, $F(3,4)=17.18, p<.01$, and there was Interlandmark Distance $\times$ Line Type interaction.

We carried out a similar analysis for the quarter group. The distance between search location and the rotated line connecting the landmarks was compared with the distance between search location and whichever NS line was closest. The means did not differ $(M=4.73, S E=1.60$, and $M=6.96, S E=0.80$, respectively), nor were the effects of interlandmark distance or the interaction of interlandmark distance and line type significant.

Triangle groups. To further analyze the effects of rotation of the landmarks on the triangle groups, the location of each of four lines was calculated. For each landmark, we calculated the location of the line based on absolute direction (the same global bearing as used during training) and the line based on relative bearing (taking into account the rotation). We then calculated the distance from the mean search location to each of the four lines. Then for each pair of lines, absolute and relative, we took the smaller distance. This gives an index of which type of bearing, relative or absolute, came closest to predicting search location. (Note that we could not use two points because the two lines defined by absolute direction did not meet anywhere near the landmarks.) These data were analyzed with a Line Type $\times$ Distance repeated measures ANOVA. The only significant effect was a general increase in the distance between search and both line types as interlandmark distance increased, $F(3,4)=5.15, p<.05$.

When the data from the constant-distance group were analyzed in this way, the results were very different from those obtained with the other groups. Search was located closer to the absolute bearings than to the relative bearings, $F(1,4)=$ $8.55, p<.05$, there was an effect of interlandmark distance, $F(3$, $4)=7.34, p<.01$, but there was no consistent increase or decrease as interlandmark distance increased. There was also a Line Type $\times$ Distance interaction, $F(3,4)=3.41, p<.05$. This interaction was due to a decrease in the use of relative bearings as interlandmark distance increased.

\section{Large Rotation}

The pattern of searching when the landmarks were rotated $90^{\circ}$ was quite different for the two groups trained with goal locations between the landmarks (see Figure 9). The nutcrackers in the halfway group used absolute bearings from the landmarks. They dug either south of the yellow landmark or north of the green landmark more often than would be expected by chance (16 out of 20 trials, binomial test, $p<.05)$. Although 

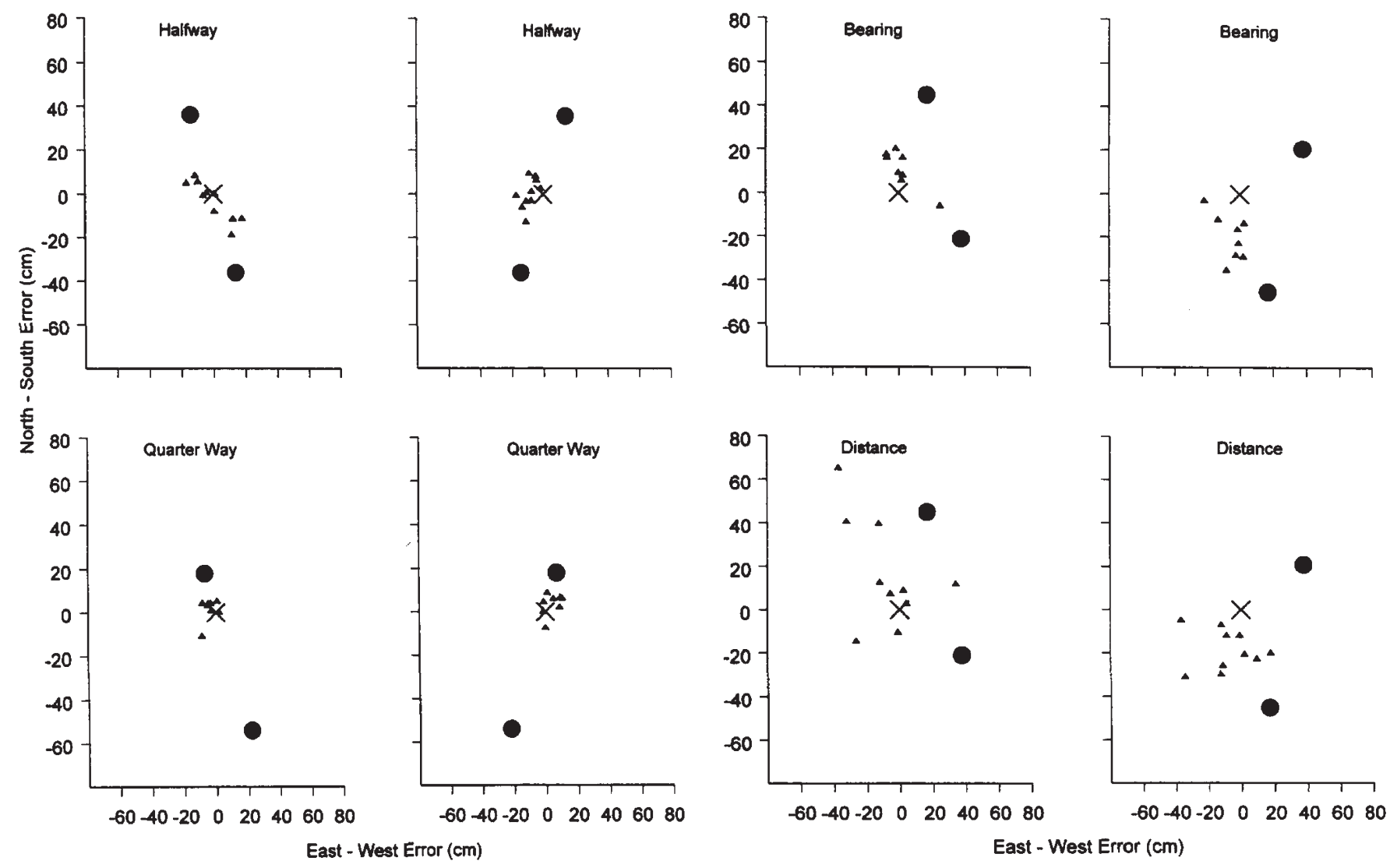

Figure 8. Maps showing the distribution of digging for each bird in each group during the clockwise (right) and counterclockwise (left) $22^{\circ}$ rotations of Experiment 3. Each triangle represents the average search location of the first five digs in a test session. The circles represent the positions of the landmarks, and the $\mathrm{X}$ marks the correct position for that group

they showed a tendency to search closer to the green than to the yellow landmark, this was not significant (14 out of 20 trials). The nutcrackers in the quarter group were even less consistent. They showed no particular tendency to dig either south of the yellow or north of the green landmark (9 out of 16 trials). They also showed no tendency to dig closer to the yellow landmark than to the green landmark (8 out of 16 trials), even though the yellow landmark was closer to the goal location throughout training.

The two triangle groups also differed. The nutcrackers in the constant-bearing group dug to the west of the array significantly more often than would be expected by chance (15 out of 16). Much of their searching behavior also appeared to be located along the lines defined by the $45^{\circ}$ bearing from the westmost landmark in the array. In contrast, search by birds in the constant-distance group was scattered, with no discernible pattern.

\section{Position Reversal (180 Rotation)}

Reversal of the landmarks had no effect on the position of the halfway point between the landmarks, and the birds in the halfway group predominantly continued to dig at the halfway point when the landmarks were rotated $180^{\circ}$ (8 out of 10 sessions, Figure 10). In the case of the quarter group, the predominant position of digging was at the nonrotated position, one quarter of the way from the green landmark (6 out of 8 sessions), although that the other 2 sessions were characterized by search near the correct rotated position. The distance and bearings groups both tended to ignore the rotation, searching to the west of the landmarks.

\section{Single-Landmark Tests}

When presented with a single landmark, the birds in both the halfway and quarter groups tended to dig along the NS axis passing through the landmark (Figure 11). Whether they dug north or south of the landmark depended on which landmark was present. For the halfway group, 10 out of 10 test sessions resulted in search north of the green landmark, and 7 out of 10 search locations were south of the yellow landmark. Thus, 17 out of 20 ( $p<.01$, binomial test) sessions led to search in the direction appropriate for the particular landmark presented. For the quarter group, search during 4 out of 8 sessions were north of the green landmark, whereas 10 out of 10 were south of the yellow landmark, so that 14 out of $18(p<.05)$ were in the appropriate direction.

All of the searching behavior of the nutcrackers in the distance and bearing groups was west of the landmark when a single landmark was presented. However, the patterns differed for the two groups. The bearing group behaved very similarly when either the green or yellow landmark was presented, with 

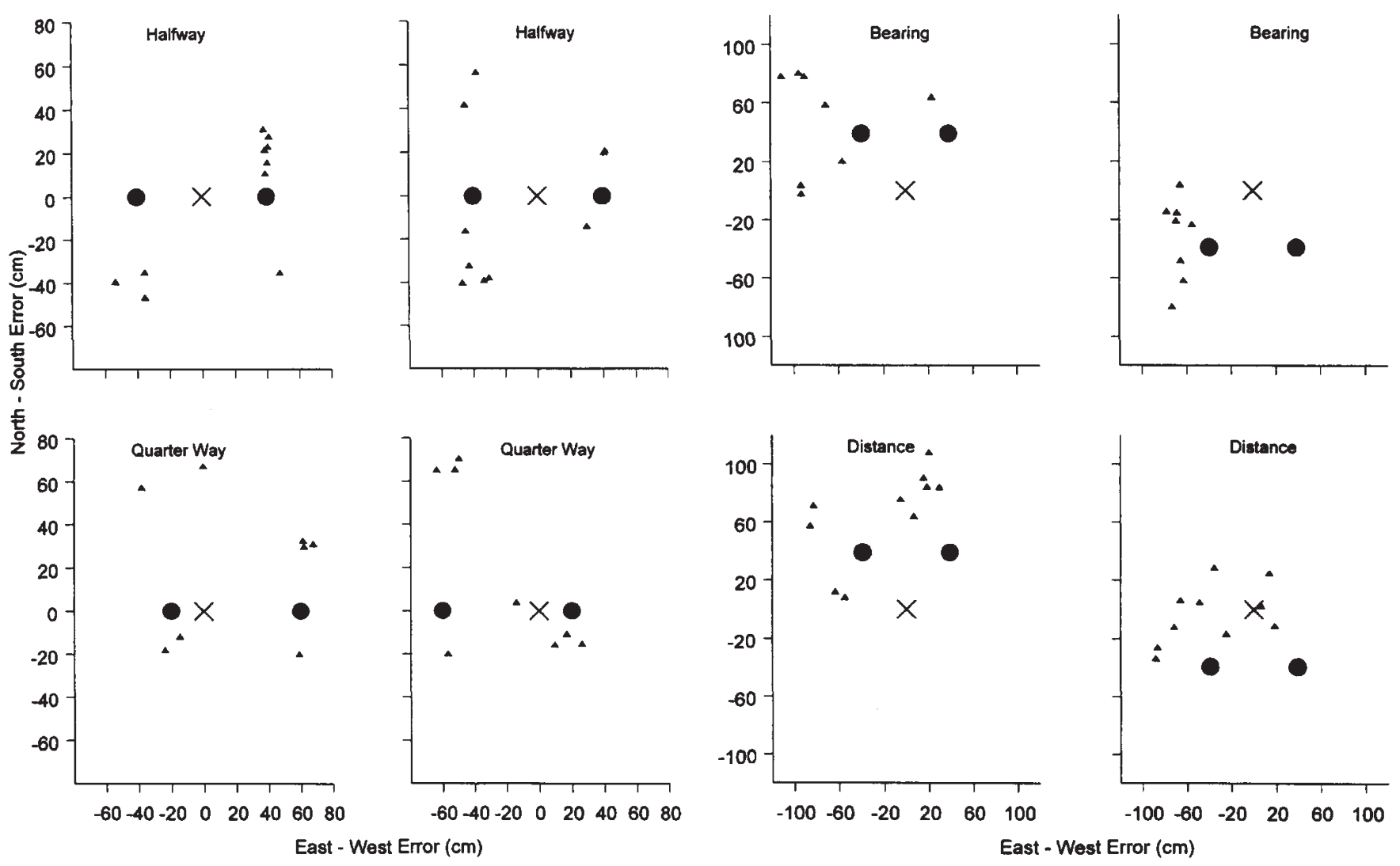

Figure 9. Maps showing the distribution of digging for each bird in each group during the clockwise and counterclockwise $90^{\circ}$ rotations of Experiment 3. Each triangle represents the average search location of the first five digs in a test session. The circles represent the positions of the landmarks, and the $\mathrm{X}$ marks the correct position for that group

14 out of $16(p<.01)$ search locations northwest of the landmark. In contrast, the bearing group tended to dig in a roughly circular arc to the west of the landmark, with location in the NS axis dependent on which landmark was presented. Searching was north of the green landmark in 9 out of 10 sessions and south of the yellow landmark during 7 out of 10 sessions. Thus, 17 out of $20(p<.01)$ sessions resulted in search in the direction appropriate for the color of the landmark being tested.

Finally, we compared the behavior of the bearing and distance groups. For each trial, we calculated the distance between the average search location and each of two predicted locations. The predicted location based on bearings was described by northwest and southwest lines radiating from the landmark. The second predicted location, based on distance, was a circle $55 \mathrm{~cm}$ in diameter centered on the landmark. A Group $\times$ Type ANOVA found a significant Group $\times$ Measure interaction, $F(1,7)=13.52, p<.01$. The bearing group searched closer to the bearing lines, whereas the distance group searched closer to the 55-cm circle.

\section{Discussion}

The results of this experiment are relevant to two issues: use of absolute versus relative bearings and the use of configural information (Cheng \& Spetch, 1998). For three groups in these experiments, the half, quarter, and bearing groups, the direc- tion between the landmarks and the goal location could be defined by either absolute or relative bearings. In terms of absolute bearings, the goal was always located in a certain compass direction from each landmark. For the half and quarter groups, this was directly south of the yellow landmark and north of the green landmark. For the bearing group, it was southwest of the yellow and northwest of the green. In terms of relative bearings for the half and quarter groups, this would be defined as long a line connecting the landmarks. For the bearing group, it would be defined as at the intersection of two lines, each with a relative bearing of $45^{\circ}$ from each landmark.

The results of the $22^{\circ}$ rotation of the landmarks from their training orientation suggest that the nutcrackers had learned both absolute and relative directional relationships between landmarks and goal. The location of search for each of the three relevant groups is clearly between the location predicted by relative and by absolute bearings. However, there is another possible interpretation. The use of absolute bearings would result in two different lines being defined when landmarks were rotated (e.g., south of the yellow and north of the green landmark would now be two different lines). It is possible that the birds were compromising between these two absolute bearings rather than between one absolute and one relative bearing. The fact that the birds in the half, quarter, and bearing groups overwhelmingly used absolute bearings when the landmarks were rotated $90^{\circ}$ lends further support to this alternative interpretation. 


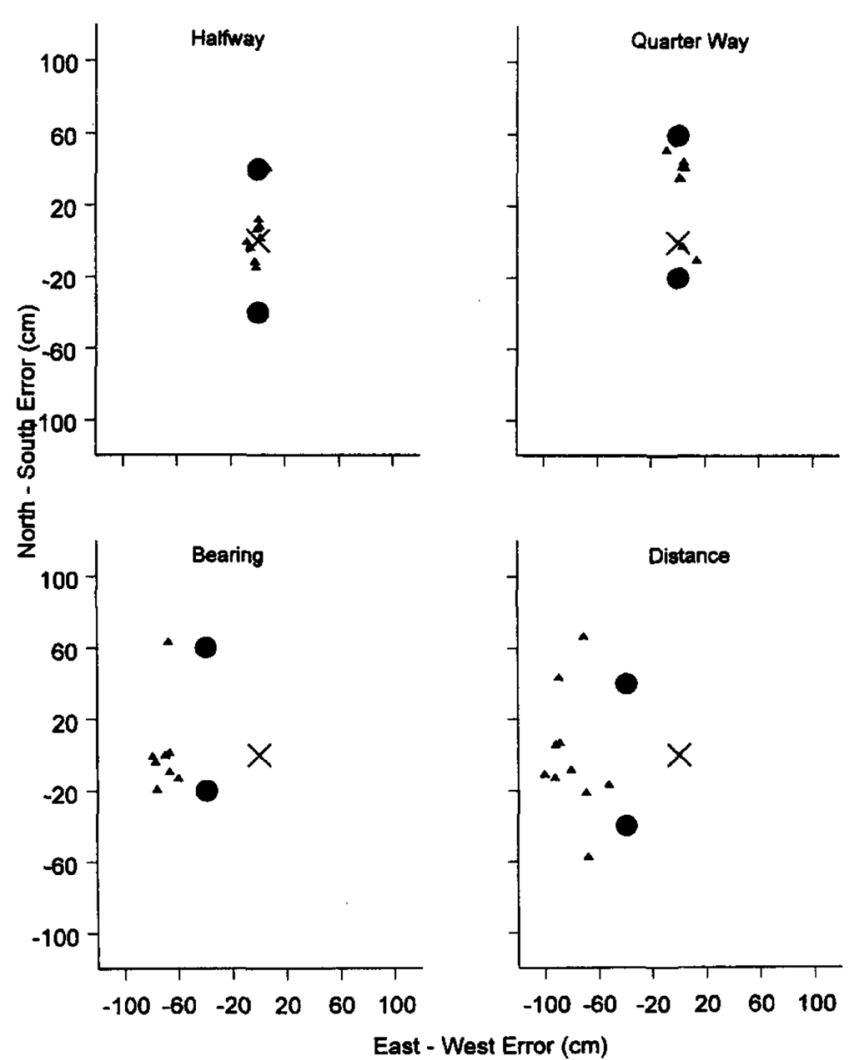

Figure 10. Maps showing the distribution of digging for each bird in each group during the clockwise and counterclockwise $180^{\circ}$ rotations of Experiment 3. Each triangle represents the average search location of the first five digs in a test session. The circles represent the positions of the landmarks, and the $\mathrm{X}$ marks the correct position for that group

The configural issue can be phrased as a question: Do the nutcrackers treat the two-landmark array as a configuration, or do they respond to the elements individually? The data from Experiment 3 make it clear that the nutcrackers do both. When the landmarks were rotated $180^{\circ}$, the position of the landmarks was reversed. If the birds were simply treating the two landmarks as an NS array, ignoring the elemental information, this reversal of position should have had no effect on the location of their search. The $180^{\circ}$ rotation data clearly show that the birds mostly ignored the rotation and therefore appear to have been treating the array as a unit or configuration.

Search behavior during the single-landmark presentations also should vary as a function of the use of configural information. If the birds were using only configural information, their behavior during single-landmark tests would be unaffected by which landmark was present. In contrast, if the birds were using elemental information, they would respond differently to the landmarks. The results clearly indicate that the direction between the single landmark and location of search for the nutcrackers in the half, quarter, and bearing groups was appropriate to the appearance of the landmark. Thus, these results support the use of elemental information. Taken together,

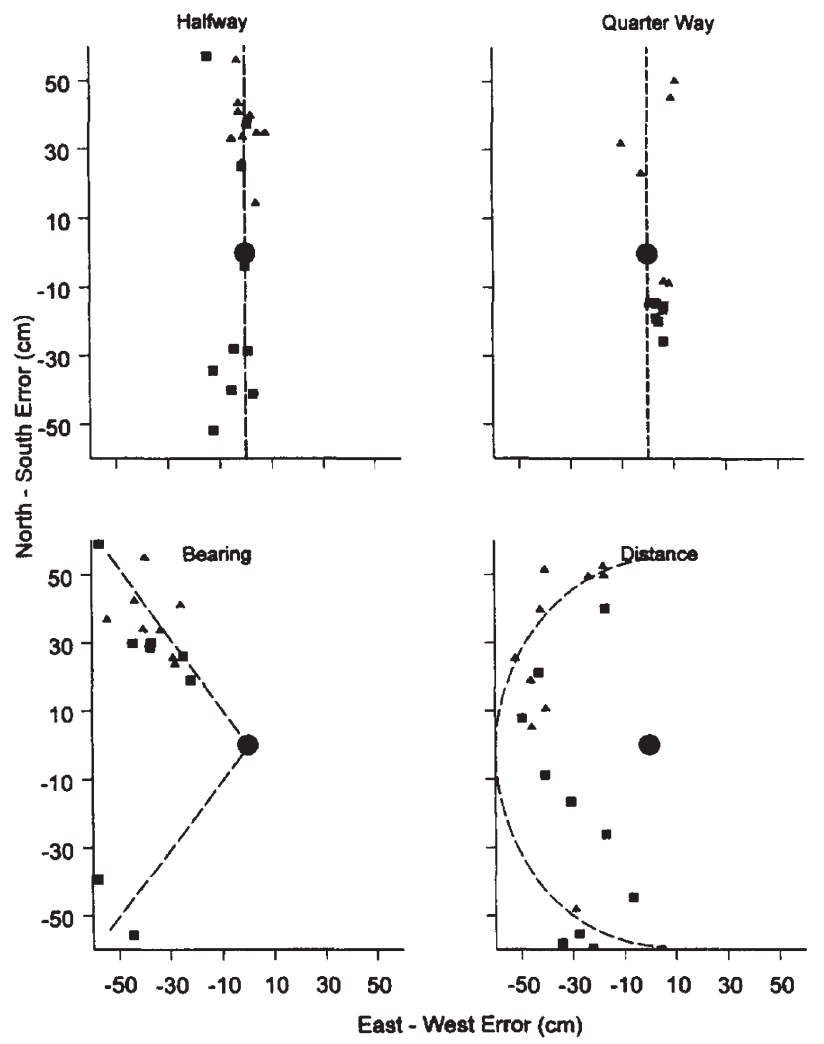

Figure 11. Maps showing the distribution of digging for each bird in each group during the single-landmark presentations of Experiment 3. Each triangle represents the average search location of the first five digs in a test session. The circle represents the position of the landmark. The dotted line represents lines described by the rules that may have been learned by each group: absolute north-south bearings for the half and quarter groups, absolute northwest and southwest bearings for the constant-bearings group, and a constant distance of $55 \mathrm{~cm}$ for the constant-distance group

then, the results of the $180^{\circ}$ rotation and of single-landmark testing show that the nutcrackers use both elemental and configural information, perhaps falling back on the elemental information when the configural information has been removed. In any event, these two types of information should not be regarded as mutually exclusive.

The results we obtained in the $180^{\circ}$ landmark rotation were exactly opposite to those obtained by Collett et al. (1986) with gerbils. This could be a species difference, but it is more likely due to differences in training protocols. In the current experiments, the nutcrackers always started from the same point, so that they always approached the landmarks from the same direction, and the room was well lit, so that other cues (which may have provided directional orientation) could be easily seen. In contrast, Collett et al. (1986)'s gerbils started from many different points, so that they approached the array from many different directions, and the room was dimly lit. This may have led the gerbils to use a different framework for bearing information. Whereas our nutcrackers maintained a consistent global orientation to the landmark array when it was reversed, the gerbils maintained a consistent relative orientation (e.g., keeping one landmark to the left and the other to the right). 


\section{General Discussion}

The abilities displayed by the nutcrackers in the quarter, half, and bearings groups throughout these experiments are reasonably characterized as geometric rule learning. The word rule is justified by the extremely precise generalization to new arrangements of the landmark array, including interlandmark distances outside of the values used during training. The word geometric is justified for two reasons. Accurate search depended on directional and/or distance information. Furthermore, when the relationships between the goal location and the landmarks are considered across all three of these groups, it is clear that the nutcrackers used multiple features of goalarray geometry, often ignoring characteristics such as absolute distance. Although it is clear that some kind of rule was learned, determining the exact nature of the rule learned by each group will require further research.

The general technique of varying landmark location so that a geometric principle defines the location of the goal provides a new tool for exploring the spatial cognitive abilities of animals. Several aspects of the results of these experiments are quite informative about the nature of spatial cognition in nutcrackers. First of all, consider what is involved in learning one of these geometric problems. What may be most impressive is not what stimuli come to control the animal's search behavior, but rather how many aspects of the situation the animal learns to ignore. Because the relevant landmarks were presented in a different location on each trial, there was no consistent relationship between the goal location and any of the other stimuli in the room. Studies with rodents have suggested that learning to use landmarks that are displaced from trial to trial but still predict the location of the goal can be quite difficult in at least some circumstances (Biegler \& Morris, 1993, 1996).

Many aspects of the relationship between the goal location and the landmarks also varied from trial to trial for the animals in these experiments. For example, for the constant-bearing group, the goal was at varying distances from the landmarks, whereas for the constant-distance group, the direction from each landmark to the goal location varied across trials. Thus, although one group learned to ignore distance and use bearing, the other group may have learned to do the opposite.

These results are very interesting in terms of Cheng's (1989) vector sum model and tests of the model (Cheng, 1994; Cheng \& Spetch, 1998). One way for an animal to code a location in terms of two landmarks is to use two vectors, each containing the direction and distance from the goal to one of the landmarks. In one series of clever experiments, Cheng (1994) attempted to differentiate between two ways of combining vectors: averaging entire vectors or averaging components of vectors. The results clearly indicated that pigeons averaged components of vectors. This suggests modularity, with the two pieces of information in the vector, distance and direction, being independently available. Our results support that idea, in that apparently nutcrackers can use either distance or direction to find a goal defined in terms of two landmarks.

Second, contrast the results of the bearing and distance groups with results from studies during which interlandmark distance was not varied during training. In several studies, subjects have been tested with varying interlandmark distances after training to find a goal centered halfway between, and a constant distance away from, two landmarks with con- stant interlandmark distance. Bossema and Pot (1974) were the first to use this technique. In their study, European jays (Garrulus glandarius) maintained the distance-direction relationship to one of the landmarks during transfer tests. Sullivan (1997) obtained similar results in a replication of Bossema and Pot (1974) with Clark's nutcrackers. Spetch et al. (1996, 1997) trained humans and pigeons with both touch screen and open-field versions of this task. Humans, but not pigeons, adjusted their vertical distance from the landmarks during probe tests, preserving the directional relationship between landmarks and the search location. The pigeons showed unsystematic variation in search location but did not change the vertical distance. However, visual inspection of their results (Spetch et al., 1997, Figure 4) suggests the pigeons may have been maintaining distance and direction from one of the landmarks. None of these studies produced results similar to those we have obtained by training nutcrackers with varying interlandmark distances (except for the results of Spetch et al., 1996, 1997, with humans).

Spetch et al. (1997), considering the ecological sense of their results with pigeons, have speculated that "it is difficult to imagine a natural situation in which it would be beneficial for an animal to match the shape of a landmark configuration but not distance of the landmarks to the goal" (p. 23). If the shape of a landmark configuration refers to the directional relationships among landmarks as seen when standing within or near the landmark array, this conclusion is false. The shape of the array changes as one's position changes. Our current results suggest that when nutcrackers use the information from a landmark array, directional information is preferred to distance information. In nature, animals may use multiple landmarks. When one possesses distance and bearing information for two or more landmarks, there is redundancy. If one knows the bearing from a goal location to each of two or more landmarks, this information is sufficient to find the goal. And if directional information is more accurate, it will be preferred.

This would seem to be particularly true when great precision is required to find the goal. This makes eminent ecological sense for Clark's nutcrackers, whose biological success requires recovering thousands of buried seed caches. These caches are distributed across wide areas, such as alpine meadows. Many of these caches are located far from any landmark that could serve as a beacon. Thus, these birds regularly face the problem of locating a goal based on relatively distant landmarks. Furthermore, given that they are searching for a fairly small target with a small shovel, they need to be very precise in their search to succeed. Thus, we would expect them to use the most accurate information available. As marine navigators well know (Bowditch, 1976), the most accurate fixes of position are obtained when the intersection of two bearings are used. Thus, we would argue that it makes excellent ecological sense for nutcrackers to use directional information to find a hidden goal.

Two aspects of the results of the current experiments suggest that directional information is more salient for nutcrackers than is distance information. The first indication comes from the data of the half and quarter groups. For these groups, given that the landmarks were located north and south of each other, the problem of placement in the EW axis was a directional problem, whereas NS placement was a distance prob- 
lem. The findings that error distances for the half group tended to be greater in the NS axis than in the EW axis and tended to increase more rapidly as interlandmark distance increased for both groups (see also Kamil \& Jones, 1997) suggest that distance information was more difficult to use than directional information. The second indication comes from the data of the triangle groups. The animals using bearings learned more rapidly and more accurately than those using distance and showed better transfer to new interlandmark distances. This also suggests that directional information is more useful than distance information.

There is no doubt that animals also use distance information in locating a goal, and the performance of the constantdistance group shows that distance information alone can be used, at least to some extent. However, when multiple landmarks are used, directional information alone can define a location. The performance of the constant-bearing group shows that nutcrackers can use bearings alone to find a goal, even when distance varies. This suggests that in many situations, directional information is more heavily weighted than distance information.

The relatively poor performance of the constant-distance group has another implication. Nutcrackers cannot solve all geometric problems with equal facility. This suggests that if we use a battery of tests, each of which assesses an animal's ability to learn a different geometric relationship, a pattern will result in which some are easily learned, others learned with difficulty, and, undoubtedly, some not learned at all. This, in turn, may provide important information about which attributes of the pattern of spatial relationships are most important to an animal's spatial orientation. This information, in turn, will reveal important information about how nutcrackers can represent spatial relationships.

\section{References}

Balda, R. P. (1980). Recovery of cached seeds by a captive Nucifraga caryotactes.. Zeitschrift fur Tierpsychologie, 52, 331-346.

Bennett, A. T. D. (1993). Spatial memory in a food-storing corvid: I. Near tall landmarks are primarily used. Journal of Comparative Physiology A, 173, 193-207.

Biegler, R., McGregor, D., \& Healy, S. D. (1998). How do animals “do" geometry? Animal Behaviour, 57, F4-F8.

Biegler, R., \& Morris, R. G. M. (1993). Landmark stability is a prerequisite for spatial but not discrimination learning. Nature, 361, 631-633.

Biegler, R., \& Morris, R. G. M. (1996). Landmark stability: Studies exploring whether the perceived stability of the environment influences spatial representation. Journal of Experimental Biology, 199, 187-193.

Bossema, I., \& Pot, W. (1974). Het terugvinden van verstopt voedsel door de Vlaamse gaai (Garrulus g. glandarius L. ) [ The recovery of stored food by Flemish jays (Garrulus g. glandarius L.)]. De Levende Natuur, 77, 265-279.
Bowditch, N. (1976). Bowditch for yachtsmen: Piloting. New York: McKay.

Cheng, K. (1986). A purely geometric module in the rat's spatial representation. Cognition, 23, 149-178.

Cheng, K. (1989). The vector sum model of pigeon landmark use. Journal of Experimental Psychology: Animal Behavior Processes, 15, 366-375.

Cheng, K. (1994). The determination of direction in landmark-based spatial search in pigeons: A further test of the vector sum model. Animal Learning and Behavior, 22, 291-301.

Cheng, K., \& Spetch, M. L. (1998). Mechanisms of landmark use in mammals and birds. In S. Healy (Ed.), Spatial representation in animals (pp. 1-18). Oxford, England: Oxford University Press.

Collett, T. S., Cartwright, B. A., \& Smith, B. A. (1986). Landmark learning and visuospatial memories in gerbils. Journal of Comparative Physiology A, 158, 835-851.

Gallistel, C. R. (1990). The organization of learning. Cambridge, MA: MIT Press.

Gould-Beierle, K., \& Kamil, A. C. (1996). The use of local and global cues by Clark's nutcrackers (Nucifraga columbiana). Animal Behaviour, 52, 519-528.

Gould-Beierle, K. L., \& Kamil, A. C. (1998). The use of landmarks in three species of food-storing corvids. Ethology, 104, 361-378.

Gould-Beierle, K. L., \& Kamil, A. C. (1999). The role of proximity in landmark use by Clark's nutcrackers. Animal Behaviour, 58, 477-488.

Kamil, A. C., Balda, R. P., Olson, D. J., \& Good, S. (1993). Revisits to emptied cache sites by Clark's nutcrackers (Nucifraga columbiana): A puzzle revisited. Animal Behaviour, 45, 241-252.

Kamil, A. C., \& Jones, J. J. (1997). The seed-storing corvid: Clark's nutcrackers learn geometric relationships among landmarks. Nature, 390, 276-279.

Kamil, A. C., \& Jones, J. E. (1998). How do they, indeed? A reply to Biegler et al. Animal Behaviour, 57, F9-F10.

Kelly, D. M., Spetch, M. L., \& Heth, C. D. (1998). Pigeons' (Columba livia) encoding of geometric and featural properties of a spatial environment. Journal of Comparative Psychology, 112, 259-269.

O'Keefe, J., \& Burgess, N. (1996). Geometric determinants of the place fields of hippocampal neurons. Nature, 381, 425-428.

Spetch, M. L., Cheng, K., \& MacDonald, S. E. (1996). Learning the configuration of a landmark array: I. Touch screen studies with pigeons and humans. Journal of Comparative Psychology, 110, 55-68.

Spetch, M. L., Cheng, K., MacDonald, S. E., Linkenhoker, B., Kelly, D., \& Doerkson, S. (1997). Use of landmark configuration by pigeons and humans: II. Generality across search tasks. Journal of Comparative Psychology, 111, 14-24.

Sullivan, B. J. (1997). The response of Clark's nutcrackers to landmark manipulations when searching for a hidden goal Unpublished master's thesis, University of Nebraska, Lincoln.

Tommasi, L., Vallortigara, G., \& Zanforlin, M. (1997). Young chickens learn to localize the centre of a spatial environment. Journal of Comparative Physiology A, 180, 567-572.

Vallortigara, G., Zanforlin, M., \& Pasti, G. (1990). Geometric modules in animals' spatial representations: A test with chicks (Gallus gallus domesticus ). Journal of Comparative Psychology, 104, 248-254. 